\title{
Ivy Sign in Moyamoya Disease: A Comparative Study of the FLAIR Vascular Hyperintensity Sign Against Contrast-Enhanced MRI
}

\author{
(D) L.-X. Wang, (DH. Wang, (D)F.-B. Hao, (D).-H. Lv, (D).-H. Zhang, (DD.-S. Han, (D)X.-B. Bian, (DD.-K. Zhang, (D).-N. Lan,
}

(D) X.-R. Wang, (D) M.-T. Wei, (D)L. Duan, (DL. Ma, and (D) X. Lou

O- $\equiv$

\begin{abstract}
BACKGROUND AND PURPOSE: The ability of the ivy sign on contrast-enhanced TT-weighted MR imaging (CEMR) to reflect cerebral perfusion and postoperative revascularization in Moyamoya disease remains largely unknown. We aimed to compare the capabilities of CEMR and FLAIR.
\end{abstract}

MATERIALS AND METHODS: CEMR, FLAIR, arterial spin-labeling, and DSA were performed in 44 patients with Moyamoya disease. The ivy sign was scored separately on CEMR and FLAIR using the Alberta Stroke Program Early CT Score. The status of leptomeningeal collaterals was scored on DSA. The postoperative Matsushima grade was evaluated at least 3 months after surgical revascularization.

RESULTS: Scoring of the ivy sign on CEMR showed excellent interrater reliability, and FLAIR vascular hyperintensity showed moderate interrater reliability. Correlation analyses revealed that DSA scores were more consistent with the CEMR-based ivy sign score $(r=0.25, P=.03)$ than with FLAIR vascular hyperintensity $(r=0.05, P=.65)$. The CEMR-based ivy sign score was significantly correlated with CBF in late-Suzuki stage Moyamoya disease $(t=-2.64, P=.02)$. The CEMR-based ivy sign score at baseline was significantly correlated with the postoperative Matsushima grade $(r=0.48, P=.03)$.

CONCLUSIONS: In this study, CEMR outperformed FLAIR in capturing the ivy sign in Moyamoya disease. In addition, the CEMR-based ivy sign score provided adequate information on hemodynamic status and postoperative neovascularization. The current study suggested that CEMR could be considered as an alternative to FLAIR in future studies investigating leptomeningeal collaterals in Moyamoya disease.

ABBREVIATIONS: CEMR = contrast-enhanced TT-weighted MR imaging; FVH = FLAIR vascular hyperintensity; MMD $=$ Moyamoya disease; PCA = posterior cerebral artery; EDAS = encephaloduroarteriosynangiosis; FOV = field of view

M oyamoya disease (MMD) is an uncommon cerebrovascular disease characterized by chronic progressive occlusion of the terminal portion of the internal carotid artery and its main branches within the circle of Willis. ${ }^{1,2}$ In MMD, the perfusion of brain tissue originates from the narrowed ICA, basal moyamoya vessels, leptomeningeal collaterals derived chiefly from the posterior circulation, and transdural collaterals from the external carotid

Received June 22, 2020; accepted after revision November 3.

From the Medical School of Chinese PLA (L.-X.W., S.-H.Z., D.-S.H.), Beijing, China; Department of Radiology (L.-X.W., J.-H.L., S.-H.Z., D.-S.H., X.-B.B., D.-K.Z., Y.-N.L., X.-R.W., M.-T.W., X.L., L.M.), the First Medical Center, Chinese PLA General Hospital, Beijing, China; and Department of Neurosurgery (H.W., F.-B.H., L.D.), the Fifth Medical Center, Chinese PLA General Hospital, Beijing, China.

L.-X. Wang, H. Wang, and F.-B. Hao contributed equally to this work. This work was supported by the National Natural Science Foundation of China (No. 81730048, 81825012).

Please address correspondence to Dr. Lin Ma, Department of Radiology, the First Medical Center of Chinese PLA General Hospital, No. 28 Fuxing Rd, Haidian District, Beijing 100853, China; e-mail: cjr.malin@vip.163.com

- Indicates open access to non-subscribers at www.ajnr.org

Indicates article with online supplemental data.

http://dx.doi.org/10.3174/ajnr.A7010 arteries. As MMD progresses, leptomeningeal collaterals remain some of the most important sources of blood supply.

Leptomeningeal collateral flow appears as high signal intensity in the subarachnoid space on contrast-enhanced T1-weighted MR imaging (CEMR) and FLAIR images. Because of its characteristic appearance (resembling ivy creeping on stone), this radiologic sign is known as the ivy sign. ${ }^{3}$ On CEMR, the ivy sign is manifested as leptomeningeal enhancement, which can decrease after bypass surgery, supporting the hypothesis that the enhancement represents the fine vascular network over the pial surface. ${ }^{4}$ Another imaging feature characterizing the ivy sign, namely, FLAIR vascular hyperintensity (FVH), has been extensively studied, especially in acute ischemic cerebrovascular diseases. In recent years, FVH has been introduced into MMD studies and was reported to be related to clinical severity and hemodynamic status in prior literature. ${ }^{5,6}$ However, a previous study reported that the ivy sign was captured better by CEMR than by FLAIR, ${ }^{7}$ raising questions of whether CEMR outperforms FLAIR as a quantitative reflection of leptomeningeal collaterals for estimating cerebral perfusion and predicting the status of postoperative neovascularization in MMD. 


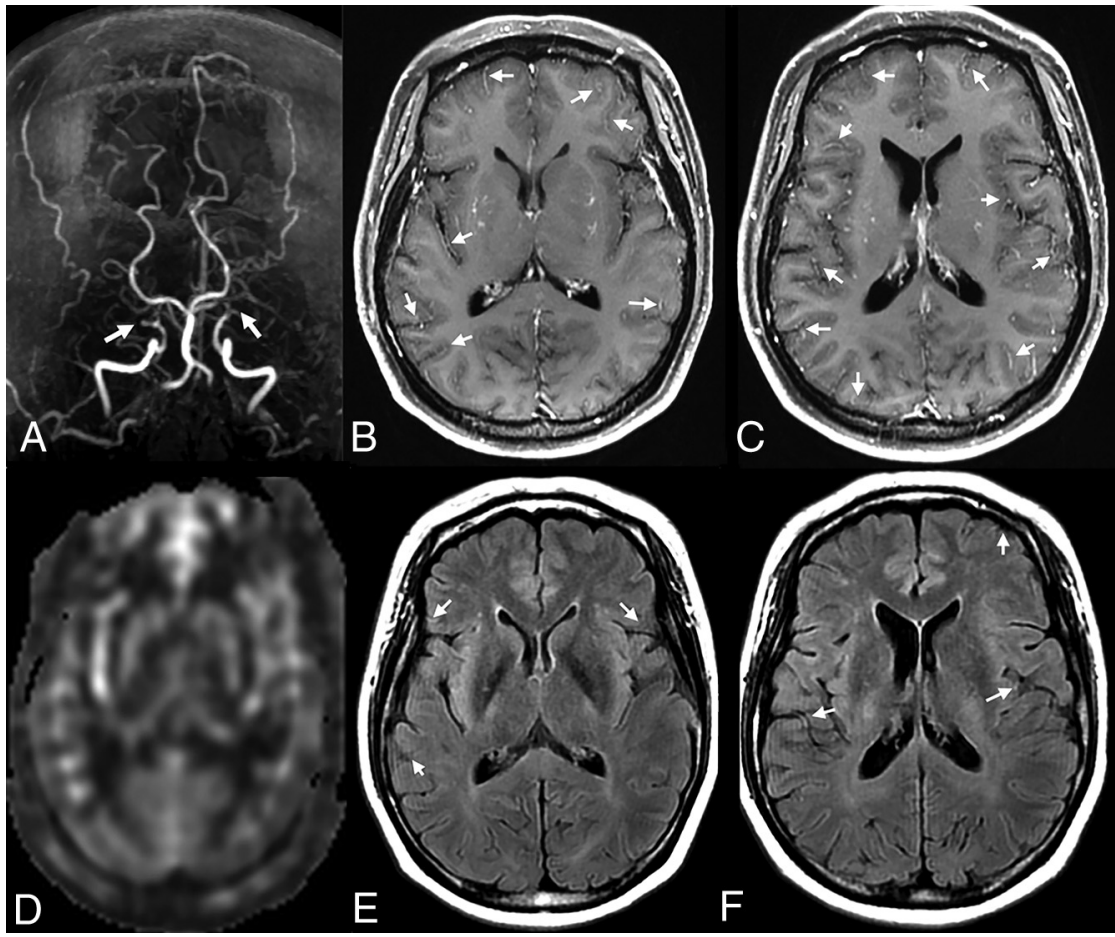

FIG 1. Illustrative case of a 45-year-old woman with bilateral MMD. MRA reveals occlusion of bilateral MCA (white arrow) (A). CEMR shows better delineation of the ivy sign than FVH (white arrows) ( $B$ and $C, E$ and $F$ ). The CBF of bilateral hemisphere shows no difference $(D)$.

carcinoma and immune system diseases. Ten patients had suspected family histories of MMD in that their relatives had ischemic or hemorrhagic stroke but had not received a definite diagnosis of MMD.

\section{Imaging Acquisition}

All subjects were scanned on a 3T MR scanner with an 8-channel phasedarray head coil.

Detailed parameters of CEMR, T2 FLAIR, high-resolution T1-weighted structural imaging, 3D pseudo-continuous arterial spin-labeling, DWI, and MRA were as follows- 1) CEMR: TR $=1850 \mathrm{~ms}, \mathrm{TE}=24 \mathrm{~ms}$, flip angle $=$ $111^{\circ}$, field of view $(\mathrm{FOV})=240 \times 240$ $\mathrm{mm}$, matrix $=320 \times 256$, slice thickness $=6 \mathrm{~mm}$, number of slices $=20$, contrast medium administration $=14$ $\mathrm{ml}(2.5 \mathrm{ml} / \mathrm{s})$, acquisition time $=1 \mathrm{mi}-$ nute 49 seconds; 2) T2 FLAIR: TR = $8500 \mathrm{~ms}, \mathrm{TE}=162 \mathrm{~ms}$, flip angle $=$ $111^{\circ}, \mathrm{FOV}=240 \times 240 \mathrm{~mm}$, matrix $=$ $288 \times 224$, slice thickness $=6 \mathrm{~mm}$, number of slices $=20$, acquisition time $=1$ minute 43 seconds; 3 ) high-re-

In this study, we compared the ability of CEMR and FLAIR to quantify the leptomeningeal ivy sign using DSA as a reference. We further evaluated the ability of the CEMR-based ivy sign score to reflect deficient perfusion in MMD and predict the postoperative Matsushima grade.

\section{MATERIALS AND METHODS \\ Patients}

This study was approved by the institutional ethics committee and registered as a clinical trial (NCT03785171). Written informed consent was obtained from each patient or his or her legally authorized representative.

From September 2018 to February 2019, this cross-sectional, prospective study enrolled 44 adult patients who were diagnosed with MMD according to the Japanese guidelines published in 2012, with the exclusion of moyamoya syndrome. ${ }^{2}$ All patients received MR imaging and DSA examinations at baseline (DSA was performed within 2 weeks after MR imaging examination). Encephaloduroarteriosynangiosis (EDAS) was performed in 34 patients (57 hemispheres) within 2 weeks after DSA examination. At least 3 months after surgery, 12 patients ( 21 hemispheres) again underwent DSA examination (the other patients had not met the 3month requirement before the data were analyzed). The exclusion criteria were as follows: 1) massive cerebral infarction or hemorrhage that could not be evaluated by MR imaging; 2) patients who had previously undergone bypass surgery with direct or indirect revascularization (indirect revascularization consists of encephalomyosynangiosis, EDAS, and encephaloduroarteriomyosynangiosis); and 3) patients with a history of systemic diseases, such as terminal solution T1-weighted structural image: TR $=6 \mathrm{~ms}, \mathrm{TE}=2.5 \mathrm{~ms}$, flip angle $=15^{\circ}, \mathrm{FOV}=256 \times 256 \mathrm{~mm}$, matrix $=256 \times 256$, slice thickness $=1 \mathrm{~mm}$, number of slices $=148$, acquisition time $=2$ minutes 24 seconds; 4) 3D pseudo-continuous arterial spin-labeling: $\mathrm{TR}=4844 \mathrm{~ms}$, $\mathrm{TE}=10.5 \mathrm{~ms}$, labeling duration $=1500 \mathrm{~ms}$, postlabeling delay $=2000 \mathrm{~ms}$, FOV $=240 \times 240 \mathrm{~mm}$, slice thickness $=$ $4.0 \mathrm{~mm}$, number of slices $=36$, acquisition time $=3$ minutes 33 seconds; 5) DWI: TR $=3000 \mathrm{~ms}, \mathrm{TE}=67.8 \mathrm{~ms}$, b-value $=1000$, FOV $=240 \times 240 \mathrm{~mm}$, slice thickness $=6 \mathrm{~mm}$, number of slices $=$ 20, acquisition time $=24$ seconds; 6 ) MRA: $\mathrm{TR}=34 \mathrm{~ms}, \mathrm{TE}=2.1$ $\mathrm{ms}, \mathrm{FOV}=240 \times 240 \mathrm{~mm}$, slice thickness $=1.4 \mathrm{~mm}$, acquisition time $=2$ minutes 32 seconds.

DSA data were collected after contrast injection in both internal carotid arteries, both external carotid arteries, and 1 or 2 vertebral arteries. Lateral and anteroposterior views of each artery injection were collected.

\section{Imaging Assessment}

MRI Assessment at Baseline. The ivy sign was defined on both CEMR and FLAIR images as continuous linear or punctate regions of leptomeningeal high signal intensity along the cortical sulci and subarachnoid space (Fig 1). Two radiologists, each with 2 years of experience, reviewed all CEMR and FLAIR images independently at 2 levels of the cerebral hemisphere (ganglionic and supraganglionic levels). The mean value was used as the final score for statistical analysis. We modified ASPECTS (Alberta Stroke Program Early CT Score, a semiquantitative method for estimating infarct size on CT) by omitting the deeper cerebral structures because they were not relevant to the assessment of 
leptomeningeal collaterals (only the M1-M6 cortical regions were included). Thus, each hemisphere was divided into 6 regions (3 regions for each level). The ivy sign score of each region was defined as follows: 0 represents no ivy sign, 1 represents the ivy sign reaching less than half of the region, and 2 represents the ivy sign reaching more than half of the region. The total ivy sign score of each hemisphere was defined as the sum score of 6 regions. 8 , 9

The DWI-positive or DWI-negative (presence of hyperintense lesion on DWI was defined as positive) status of bilateral MCA territory was recorded at baseline and was used to indicate whether cerebral lesions in the acute or subacute phase existed. DWI features were used to investigate whether the ivy sign was related to the time from stroke onset to imaging (which was reflected by the DWI signal).

MCA stenosis was rated on MRA by 1 radiologist and defined as moderate to severe stenosis (50\%-99\%). MCA occlusion was recorded when no visible blood flow could be seen on MRA images.

DSA Assessment at Baseline. The DSA score was defined as follows: leptomeningeal collateral assessment on DSA was evaluated according to Liu et $\mathrm{al}^{10}$ by 2 experienced neurosurgeons. This leptomeningeal scoring system ranges from 0 to 6 and includes 3 parts of the collateral networks: pPCA $\rightarrow$ ACA (the parieto-occipital branch of the posterior cerebral artery (PCA) anastomoses to the ACA), tPCA $\rightarrow \mathrm{tMCA}$ (the anterior temporal branch of the posterior cerebral artery anastomoses to the temporal branch of the MCA), and pPCA $\rightarrow$ MCA (the pPCA anastomoses to the MCA). Additionally, transdural collaterals were assessed and scored ( 0 represents the absence of transdural collaterals, and 1 represents the presence of transdural collaterals). The total DSA score was defined as the sum of leptomeningeal collaterals and transdural collaterals and was considered the reference standard in this study.

The Suzuki stage, a well-known cerebral angiographic classification for MMD, was also recorded. In this study, we defined the late-Suzuki stage group as Suzuki stage $\geq$ IV and the earlySuzuki stage group as Suzuki stage $\leq$ III for further subgroup analysis.

DSA Assessment at Follow-up. As stated earlier, postoperative follow-up DSA examinations were valid for 12 patients (21 hemispheres). At least 3 months after EDAS, the 21 hemispheres were graded postoperatively according to the following system proposed by Matsushima et $\mathrm{al}^{11}$ : grade I was defined as revascularization of less than one-thirds of the MCA distribution, grade II as revascularization of one-thirds to two-thirds of the MCA distribution, and grade III as revascularization of more than two-thirds of the MCA distribution. The Matsushima score was used to assess the effect of revascularization after EDAS.

\section{Quantitative Estimation of CBF}

Quantitative estimation of the CBF of the MCA territory was performed automatically. First, the CBF map of 3D pseudo-continuous arterial spin-labeling was processed and generated using FuncTool (AW 4.5 Workstation, GE Healthcare). Second, high- resolution T1 structural images were normalized to Montreal Neurological Institute space using FLIRT (FMRIB Linear Image Registration Tool; https://fsl.fmrib.ox.ac.uk/fsl/fslwiki/FLIRT) and FNIRT (FMRIB Non-Linear Image Registration Tool; https://fsl.fmrib.ox.ac.uk/fsl/fslwiki/FNIRT) within the FMRIB Software Library (FSL, http://www.fmrib.ox.ac.uk/fsl). Then CBF maps were affine registered to the normalized T1 structural images using FLIRT. ${ }^{12}$ Next, a brain mask of the MCA territory was projected to the normalized CBF maps, as described elsewhere. ${ }^{13}$ Finally, the mean CBF of the MCA territory of each hemisphere was extracted and prepared for further statistical analysis.

\section{Statistical Analysis}

The interrater reliability of scoring was evaluated by calculating the intraclass correlation coefficient (ICC). ICC values range from 0 to 1 , and a value above 0.75 was considered to indicate excellent reliability. ${ }^{14}$ The correlations of the CEMR and FLAIR ivy sign scores with DSA were examined by Pearson correlation analysis. The relationships of these 2 scoring modalities with Suzuki stage were assessed using Spearman correlation analysis. A 2-tailed independent Student $t$ test was performed on the CEMR and FLAIR ivy signs to differentiate between MCA stenosis and MCA occlusion. Linear regression was used to evaluate the statistical significance of these 2 scoring modalities in reflecting the CBF of each hemisphere. Because the PCA is a major source of blood supply for leptomeningeal collaterals and the involvement of the PCA may greatly affect cerebral perfusion, PCA involvement was included as a covariant. Other potentially relevant covariates included sex, age, and illness duration. A subgroup analysis was performed in patients with MMD in late versus early Suzuki stages using the same method. Ivy sign scores based on CEMR and FLAIR were compared between DWI-positive and DWI-negative cases using a 2-tailed independent Student $t$ test. Partial correlation analysis was used to determine the relationship between imaging parameters and Matsushima grades, with PCA involvement included as a covariate. $P<.05$ was considered to indicate statistical significance. All statistical analyses were performed using SPSS 22.0 (IBM).

\section{RESULTS}

\section{Demographic Characteristics}

A total of 44 patients with MMD (including 9 patients with unilateral MMD and 35 patients with bilateral MMD, for a total of 79 hemispheres) were recruited in the current study. The demographic and clinical characteristics of the enrolled patients are shown in Table 1.

\section{Consistency of CEMR-Based Ivy Sign Score and FVH with DSA Scores}

There was excellent interrater reliability of CEMR-based ivy sign score and DSA scores. FVH showed moderate interrater reliability (Table 2).

Correlation analysis revealed a statistically significant relationship between CEMR-based ivy sign scores and DSA scores $(r=0.25, P=.03)$ but not between FVH and DSA scores $(r=0.05, P=.65)$. Regarding the correlations between these 2 
Table 1: Demographic characteristics and clinical measurements of patients with MMD

Left Hemisphere $(n=38) \quad$ Right Hemisphere $(n=41)$

\begin{tabular}{l}
\hline Age (years) \\
Sex (male/female) \\
Illness duration (months) \\
Family history (y/n) \\
Initial manifestation on imaging \\
Ischemic onset \\
Hemorrhagic onset \\
DWI (positive/negative) \\
MCA (stenosis/occlusion) \\
PCA (normal/stenosis/occlusion) \\
Mean Suzuki stage \\
CBF of MCA territory (mL/100 \\
g/min) \\
Scoring of leptomeningeal \\
collaterals \\
FVH \\
CEMR-based ivy sign score \\
DSA score
\end{tabular}

$41.63 \pm 12.31$
$18 / 26$
$45.85 \pm 76.76$
$3 / 31$

$\begin{array}{lc}11 & 6 \\ 3 & 9 \\ 7 / 31 & 3 / 38 \\ 11 / 27 & 8 / 33 \\ 25 / 8 / 5 & 29 / 8 / 4 \\ \text { IV }(I I-V) & \text { IV (III-V) } \\ 47.52 \pm 11.39 & 46.50 \pm 15.43\end{array}$

$2.91 \pm 1.59$

$7.87 \pm 2.18$

$2.63 \pm 1.84$
$2.68 \pm 1.52$

$7.55 \pm 2.20$

$2.65 \pm 1.88$

Note:-Continuous data were presented as mean \pm SD, and categorical data were presented as median (IQR).

Table 2: Interrater reliability of FVH, CEMR-based ivy sign score, and DSA score

\begin{tabular}{lccc}
\hline & & \multicolumn{2}{c}{$95 \% \mathrm{Cl}$} \\
\cline { 3 - 4 } & ICC & Lower Bound & Upper Bound \\
\hline FVH & 0.71 & 0.55 & 0.82 \\
CEMR-based ivy sign score & 0.81 & 0.70 & 0.88 \\
DSA score & 0.83 & 0.73 & 0.89 \\
\hline
\end{tabular}

imaging modalities and Suzuki stage, the CEMR-based ivy sign score showed a significant correlation $(\rho=0.44, P<.001)$. For $\mathrm{FVH}$, although a significant correlation was found, the correlation coefficient was lower than that of the ivy sign on CEMR $(\rho=0.24, P=.03)($ Fig 2$)$.

Patients with DWI-positive and DWI-negative MMD had significantly different ivy sign scores based on FLAIR $(t=2.82$, $P=.01)$ but not on CEMR $(t=-0.19, P=.85)$ (Online Supplemental Data).

\section{CEMR-Based Ivy Sign Score and FVH for Differentiating the Severity of MCA Stenosis}

The CEMR-based ivy sign score outperformed FVH (FVH, $t=$ $-1.73, P=.09$; CEMR-based ivy sign score, $t=-2.32, P=.03$; DSA, $t=-3.01, P=.005)$ in differentiating between MCA stenosis and MCA occlusion.

\section{Relationships between the Ivy Sign and CBF}

High-resolution T1-weighted structural images were unavailable in 13 patients with MMD because of severe head motion or patients' reluctance to undergo the scan. A total of 31 patients' CBF (in 54 hemispheres) was quantified and included in the analyses. In stepwise linear regression analysis with the CEMR-based ivy sign score or FVH, involvement of PCA, age, sex, and illness duration specified as independent variables, no variable was included in the final model for either the CEMR-based ivy sign score or FVH.

In the subgroup analyses, the early-Suzuki stage MMD group had a trend of positive correlation between CEMR-based ivy sign score and CBF, though the correlation was not statistically significant (Online Supplemental Data). In the late-Suzuki stage MMD group, a linear regression model showed that the CEMR-based ivy sign score can serve as an independent indicator for determining CBF (Table 3).

\section{Relationship between Imaging Parameters and Matsushima Grade} Partial correlation analysis demonstrated a statistically significant correlation between the CEMR-based ivy sign score and Matsushima grade $(r=0.48$, $P=.03$ ). No significant relationship was found between FVH and Matsushima grade $(r=0.17, P=.46)$.

\section{DISCUSSION}

In patients with MMD, leptomeningeal collaterals play an important role in maintaining cerebral perfusion. On MR images, these collaterals can be represented by the ivy sign. In this study, we compared $2 \mathrm{MR}$ approaches for assessing the ivy sign (ie, CEMR and FLAIR), with DSA as a reference. We found that the ivy sign on CEMR outperformed the ivy sign on FLAIR in depicting leptomeningeal collaterals, as well as in reflecting the Suzuki stage of MMD. More important, we found that the CEMR-based ivy sign score could serve as an independent biomarker for predicting $\mathrm{CBF}$ in late-Suzuki stage MMD. In addition, the CEMR-based ivy sign score can predict the status of postoperative neovascularization in patients with MMD after EDAS.

The current study found that the ivy sign was less obvious on FLAIR than on CEMR. Moreover, FVH failed to reflect the DSA score for leptomeningeal collaterals. FVH has been extensively studied in the field of atherosclerotic diseases, such as ischemic stroke. ${ }^{15}$ This radiologic sign is considered to be related to collateralization, ${ }^{16}$ stroke severity, ${ }^{17}$ and functional outcomes. ${ }^{16}$ Physically, FVH is likely to reflect slow retrograde or turbulent flow in the engorged pial collateral arteries through leptomeningeal anastomoses. ${ }^{18}$ In recent years, FVH has also been introduced into MMD studies as an indicator of collateral blood flow. With regard to acute ischemic stroke, a previous study reported a $98 \%$ prevalence of FVH in the first 6 hours after onset. ${ }^{19}$ Another study also suggested that FVH was a temporary phenomenon that commonly disappeared within the first 24-36 hours after stroke onset. ${ }^{17}$ The short-term presence of FVH raised the question of whether it is appropriate for use in MMD studies. Although conceptually comparable to MMD, the underlying mechanism of collateral formation in acute stroke and MMD may not be the same. In a chronic state of hypoperfusion, as is found in MMD, FVH was unlikely to appear as a rapid reaction as in acute stroke. In this study, DWI hyperintensity was used to indicate whether lesions in the acute or subacute phase existed, which could indirectly reflect the time from onset to imaging. We found significant differences between patients with DWI-positive and DWI-negative MMD with respect 

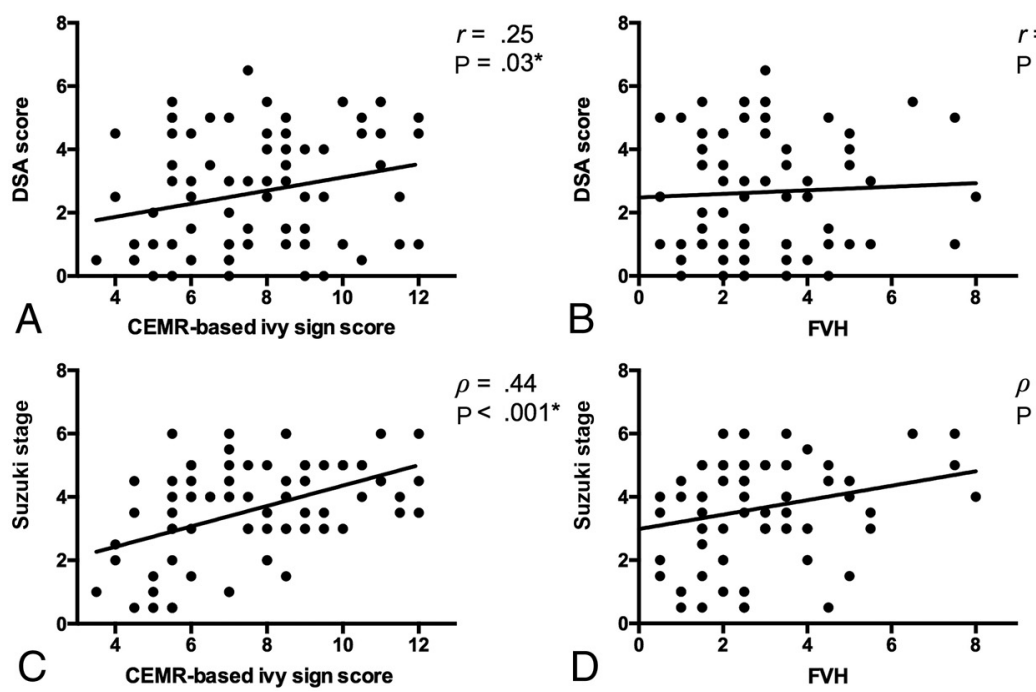

$r=.05$

$=.65$

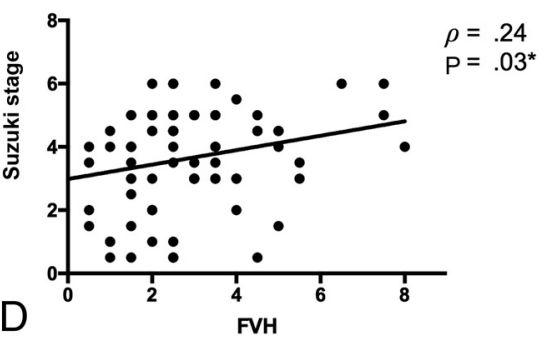

FIG 2. Scatter plots of the correlation between ivy sign score on CEMR (A), FVH (B) and DSA score of leptomeningeal collaterals; correlation between ivy sign score on CEMR (C), FVH (D) and Suzuki stage of Moyamoya disease. The asterisk indicates $P<0.05$.

Table 3: Multivariable linear regression analysis results for CEMR-based ivy sign score with $C B F$ as the dependent variable in patients with late-Suzuki stage $M_{M D}{ }^{a}$

\begin{tabular}{lccccc}
\hline & B & SE & $\boldsymbol{\beta}$ & $\boldsymbol{t}$ & $\boldsymbol{P}$ Value \\
\hline Included variable & & & & & \\
$\quad$ Constant & 65.91 & 8.49 & - & 7.77 & $<.001^{\mathrm{b}}$ \\
CEMR-based ivy sign score & -2.49 & 0.94 & -0.49 & -2.64 & $.015^{\mathrm{b}}$ \\
Excluded variable & & & & & \\
Age & - & - & -0.02 & -0.12 & .54 \\
Sex & - & - & -0.09 & -0.46 & .65 \\
Illness duration & - & - & 0.12 & 0.62 & .54 \\
PCA & - & - & -0.18 & -0.95 & .35 \\
\hline
\end{tabular}

${ }^{\mathrm{a}} F=6.95, P=.015 ; R^{2}=0.24$, adjusted $R^{2}=0.21$.

${ }^{\mathrm{b}} P<0.05$.

Note:-CEMR indicated contrast-enhanced T7-weighted MR images; MMD, Moyamoya disease; PCA, posterior cerebral artery; $\mathrm{B}$, partial regression coefficient; $\beta$, standardized partial regression coefficient; $\mathrm{SE}$, standard error.

arachnoid membranes and edema. Our results agreed with those of Yoon et $\mathrm{al}^{7}$; CEMR-based ivy sign score was higher than FVH and was significantly correlated with DSA score, but such a correlation was not found for FVH.

During the course of MMD, MCA stenosis and occlusion can appear at different stages of disease. Some unilateral MMD cases can even show a normal MCA. We included hemispheres with MCA stenosis or occlusion in this study and found that CEMR-based ivy sign scores and DSA scores could differentiate between these levels of stenotic severity, but FVH could not. Such results further supported the hypothesis that $\mathrm{FVH}$ might not be appropriate for the evaluation of leptomeningeal collaterals in patients with MMD.

Taking a step further, we studied the relationship of the CEMR-based ivy sign score and $\mathrm{FVH}$ with $\mathrm{CBF}$. FVH and CBF were found to be unrelated. A previous study by Noguchi et $\mathrm{al}^{23}$ found similar results. These investigators found that CBF showed no difference among patients with MMD with 3 levels of FVH, though a different scoring system for the ivy sign was used in their study. Mori et $\mathrm{al}^{18}$ claimed that the cerebral vasculature in the region with decreased perfusion pressure is already dilated to maintain CBF. In their study, FVH could indi-

to FVH, which added further evidence in support of this explanation. Physically, the presence of FVH is rooted mainly in slow flow via leptomeningeal collaterals, ${ }^{20}$ which may not be the case in MMD. A possible hypothesis is that the flow velocity of leptomeningeal collaterals in MMD after a relatively long illness duration was different from that in acute stroke. A previous study demonstrated obviously increased cortical microvascular attenuation and diameter in MMD compared with atherosclerotic disease. ${ }^{21}$ Such differences in the diameters, lengths, and pressures of the collateral routes may induce different flow velocities, which are related to the presence of FVH.

Previous studies have demonstrated that CEMR is superior to FLAIR in capturing the ivy sign. ${ }^{7,22}$ To our knowledge, there has been no quantitative comparison of these 2 modalities with regard to their consistency with DSA. Additionally, previous studies reached different conclusions in terms of the incidence of the ivy sign; that is, Yoon et $\mathrm{al}^{7}$ found a higher incidence of the ivy sign on CEMR than FLAIR, but Jung et $\mathrm{al}^{22}$ found the opposite phenomenon. Yoon et $\mathrm{al}^{7}$ 's finding is readily comprehensible because from a pathophysiological viewpoint, one can predict enhancement of the engorged pial vessels with thickened cate decreased cerebrovascular reserve, which suggested that the cerebrovascular reserve might be more sensitive than CBF.

We also found a nonsignificant correlation between the CEMRbased ivy sign score and CBF of the MCA territory when all cases were included in the statistical analysis. We then divided the patients with MMD into an early-Suzuki stage group and a lateSuzuki stage group. In patients with early-Suzuki stage MMD, the trend of correlation between CEMR-based ivy sign score and CBF was positive, though no statistical significance was achieved. Interestingly, a negative correlation was found between the CEMRbased ivy sign score and CBF in patients with late-Suzuki stage MMD. A possible explanation for this result was the imbalance between leptomeningeal collaterals and abnormal ICA and moyamoya vessels. We hypothesized that in the late Suzuki stages, the ability of leptomeningeal collaterals to compensate for cerebral perfusion was inadequate. However, in early-Suzuki stage MMD, the trend of a positive correlation between CEMR-based ivy sign scores and $\mathrm{CBF}$ suggested that the more collaterals there were, the higher the $\mathrm{CBF}$ was. The potentially significant correlation found between CEMR-based ivy sign scores and CBF in all cases might be covered by the inclusion of patients with early-Suzuki stage MMD. 
In an article by Liu et al, ${ }^{10}$ the grading of leptomeningeal collaterals on DSA was defined as the sum of collaterals from PCA without transdural collateral vessels. However, unlike DSA, MR imaging signs represent all possible leptomeningeal collaterals regardless of the origin of blood flow. In this study, the DSA score for leptomeningeal collaterals was defined as the sum of scores for blood flow originating from the posterior circulation and transdural vessels. The significant correlation between the CEMR-based ivy sign score and DSA score implies that the ivy sign on CEMR may represent all collaterals to brain tissue, including collaterals from the PCA and external carotid artery. The ivy sign on CEMR may have an advantage in that it provides a noninvasive visualization of leptomeningeal collaterals, including transdural collaterals.

Previous literature has reported that leptomeningeal collaterals decrease in abundance after cerebral revascularization in patients with MMD. ${ }^{24}$ Such findings suggest that leptomeningeal collaterals are associated with cerebral hemodynamics, which would improve after surgery. ${ }^{24}$ After EDAS, newly formed collaterals from the external carotid artery serve as an important source of cerebral perfusion. Thus, it is possible that leptomeningeal collaterals at baseline could predict the growth of transdural collaterals, an essential indicator of the effectiveness of EDAS. In this study, we found that Matsushima grades correlated significantly with the CEMR-based ivy sign score at baseline. The results indicated that the more leptomeningeal collaterals were present at baseline, the better the outcome of the revascularization operation would be.

Our results should be considered in the context of several limitations. First, among the 79 hemispheres studied, only 10 showed hyperintense lesions on DWI. The statistical power may be jeopardized because of the disparity between DWI-positive and DWInegative hemispheres. A similar shortage occurred in the linear regression subgroup analysis of CEMR-based ivy sign scores and CBF. The relatively small sample size of early-Suzuki stage MMD may have resulted in failure to find a significant association between these 2 variables. Second, because of the limited number of hemispheres with postoperatively evaluated Matsushima grades (only 21 hemispheres), future studies with additional subjects are needed to estimate the association between leptomeningeal collaterals at baseline and the effectiveness of surgical revascularization. Third, when contrast-enhanced MR imaging is performed, the potential long-term risks associated with gadolinium retention should be carefully considered. Finally, techniques such as deep learning may have the potential to considerably enhance the diagnostic value of the ivy sign on CEMR and FLAIR.

\section{CONCLUSIONS}

The current study concerning the application of FVH in the field of MMD suggested that the CEMR-based ivy sign score was superior to FVH in visualizing leptomeningeal collaterals, with DSA as a reference. Furthermore, the CEMR-based ivy sign score could serve as a possible independent predictor of $\mathrm{CBF}$ in late-Suzuki stage MMD and reflect the effectiveness of surgical revascularization.

Disclosures: Xin Lou—RELATED: Grant: the National Natural Science Foundation of China, Comments: No. 81730048, 81825012.

\section{REFERENCES}

1. Kuroda S, Houkin K. Moyamoya disease: current concepts and future perspectives. Lancet Neurol 2008;7:1056-66 CrossRef Medline

2. Research Committee on the Pathology and Treatment of Spontaneous Occlusion of the Circle of Willis; Health Labour Sciences Research Grant for Research on Measures for Infractable Diseases. Guidelines for diagnosis and treatment of moyamoya disease (spontaneous occlusion of the circle of Willis). Neurol Med Chir (Tokyo) 2012;52:245-66 CrossRef Medline

3. Sivrioglu AK, Saglam M, Yildiz B, et al. Ivy sign in Moyamoya disease. Eurasian J Med 2016;48:58-61 CrossRef Medline

4. Komiyama $M$, Nakajima $H$, Nishikawa $M$, et al. Leptomeningeal contrast enhancement in moyamoya: its potential role in postoperative assessment of circulation through the bypass. Neuroradiology 2001;43:17-23 CrossRef Medline

5. Kawashima M, Noguchi T, Takase Y, et al. Unilateral hemispheric proliferation of ivy sign on fluid-attenuated inversion recovery images in moyamoya disease correlates highly with ipsilateral hemispheric decrease of cerebrovascular reserve. AJNR Am J Neuroradiol 2009;30:1709-16 CrossRef Medline

6. Seo K-D, Suh SH, Kim YB, et al. Ivy sign on fluid-attenuated inversion recovery images in moyamoya disease: correlation with clinical severity and old brain lesions. Yonsei Med J 2015;56:1322-27 CrossRef Medline

7. Yoon H-K, Shin H-J, Chang YW. "Ivy sign" in childhood moyamoya disease: depiction on FLAIR and contrast-enhanced T1weighted MR images. Radiology 2002;223:384-89 CrossRef Medline

8. Horie N, Morikawa M, Morofuji Y, et al. De novo ivy sign indicates postoperative hyperperfusion in moyamoya disease. Stroke 2014;45:1488-91 CrossRef Medline

9. Barber PA, Demchuk AM, Zhang J, et al. Validity and reliability of a quantitative computed tomography score in predicting outcome of hyperacute stroke before thrombolytic therapy. ASPECTS Study Group. Alberta Stroke Programme Early CT Score. Lancet 2000;355:1670-74 CrossRef Medline

10. Liu Z-W, Han C, Zhao F, et al. Collateral circulation in Moyamoya disease: a new grading system. Stroke 2019;50:2708-15 CrossRef Medline

11. Matsushima Y, Inaba Y. The specificity of the collaterals to the brain through the study and surgical treatment of moyamoya disease. Stroke 1986;17:117-22 CrossRef Medline

12. Jenkinson M, Bannister P, Brady M, et al. Improved optimization for the robust and accurate linear registration and motion correction of brain images. Neuroimage 2002;17:825-41 CrossRef Medline

13. Lyu J, Ma N, Liebeskind DS, et al. Arterial spin labeling magnetic resonance imaging estimation of antegrade and collateral flow in unilateral middle cerebral artery stenosis. Stroke 2016;47:428-33 CrossRef Medline

14. Cho S, Yankanah R, Babyn $\mathrm{P}$, et al. Inter-rater reliability of the radiographic assessment of simple bone cysts. J Child Orthop 2019;13:22635 CrossRef Medline

15. Azizyan A, Sanossian N, Mogensen MA, et al. Fluid-attenuated inversion recovery vascular hyperintensities: an important imaging marker for cerebrovascular disease. AJNR Am J Neuroradiol 2011;32:1771-75 CrossRef Medline

16. Jiang $L$, Chen $Y-C$, Zhang $H$, et al. FLAIR vascular hyperintensity in acute stroke is associated with collateralization and functional outcome. Eur Radiol 2019;29:4879-88 CrossRef Medline

17. Hohenhaus M, Schmidt WU, Brunecker P, et al. FLAIR vascular hyperintensities in acute ICA and MCA infarction: a marker for mismatch and stroke severity? Cerebrovasc Dis 2012;34:63-69 CrossRef Medline

18. Mori N, Mugikura S, Higano S, et al. The leptomeningeal "ivy sign” on fluid-attenuated inversion recovery MR imaging in Moyamoya disease: a sign of decreased cerebral vascular reserve? AJNR Am J Neuroradiol 2009;30:930-05 CrossRef Medline 
19. Toyoda K, Ida M, Fukuda K. Fluid-attenuated inversion recovery intraarterial signal: an early sign of hyperacute cerebral ischemia. AJNR Am J Neuroradiol 2001;22:1021-29 Medline

20. Sanossian N, Saver JL, Alger JR, et al. Angiography reveals that fluidattenuated inversion recovery vascular hyperintensities are due to slow flow, not thrombus. AJNR Am J Neuroradiol 2009;30:564-68 CrossRef Medline

21. Czabanka M, Peña-Tapia P, Schubert GA, et al. Characterization of cortical microvascularization in adult moyamoya disease. Stroke 2008;39:1703-09 CrossRef Medline
22. Jung MY, Kim YO, Yoon W, et al. Characteristics of brain magnetic resonance images at symptom onset in children with moyamoya disease. Brain Dev 2015;37:299-306 CrossRef Medline

23. Noguchi T, Kawashima M, Nishihara M, et al. Arterial spin-labeling MR imaging in Moyamoya disease compared with clinical assessments and other MR imaging findings. Eur J Radiol 2013;82:e84047 CrossRef Medline

24. Kawashima M, Noguchi T, Takase $Y$, et al. Decrease in leptomeningeal ivy sign on fluid-attenuated inversion recovery images after cerebral revascularization in patients with Moyamoya disease. AJNR Am J Neuroradiol 2010;31:1713-18 CrossRef Medline 